\title{
Forest without prey: livestock sustain a leopard Panthera pardus population in Pakistan
}

\author{
Wasim Shehzad, Muhammad Ali Nawaz, François Pompanon, Eric Coissac \\ Tiayya Riaz, Safdar Ali Shah and Pierre Taberlet
}

\begin{abstract}
Human-carnivore conflict is one of the major challenges in the management of populations of large carnivores. Concerns include the increasing human population; habitat loss as a result of degradation and fragmentation of forest; and livestock predation as a result of a lack of natural prey, leading to retaliatory killings of wild carnivores. Conflicts may be further aggravated by occasional attacks that result in injury and loss of human life. The level of consumption of prey species by a predator is a benchmark to evaluate the scale of this conflict. We used a newly developed DNA-based diet analysis to study the prey profile of common leopards Panthera pardus in Ayubia National Park, Pakistan. The results suggest that the common leopard is a generalist predator, subsisting mainly on domestic animals. Based on the frequency of occurrence of prey items in 57 faecal samples, the diet of the leopard is dominated by domestic goat Capra hircus (64.9\%), followed by domestic dog Canis lupus familiaris (17.5\%) and cow Bos taurus (12.3\%). Domestic animals (goat, dog, cow, water buffalo Bubalus bubalis, horse Equus caballus and sheep Ovis aries) occurred in 54 (95\%) of the 57 samples. We recommend a two-step strategy to mitigate this conflict: (1) introducing incentives for increased acceptance of leopards among local communities in the vicinity of the protected area and (2) increasing the availability of wild prey. We hope that the results of this study will contribute to the survival of the leopard in Pakistan.
\end{abstract}

Keywords Blocking oligonucleotide, DNA metabarcoding, human-carnivore conflict, leopard, next-generation sequencing, Pakistan, Panthera pardus

This paper contains supplementary material that can be found online at http://journals.cambridge.org

Wasim ShehzaD*, François Pompanon, Eric Coissac, Tiayyba Riaz and Pierre TABERLET (Corresponding author) Laboratoire d'Ecologie Alpine, CNRS UMR 5553, Université Joseph Fourier, BP 53, F-38041 Grenoble Cedex 9, France E-mail pierre.taberlet@ujf-grenoble.fr

Muhammad Ali Nawaz Department of Animal Sciences, Quaid-i-Azam University, Islamabad, Pakistan

Safdar Ali Shah Wildlife Department, Khyber Pakhtunkhwa, Pakistan

*Also at: Institute of Biochemistry and Biotechnology, University of Veterinary and Animal Sciences, Lahore, Pakistan

Received 24 October 2012. Revision requested 11 February 2013.

Accepted 12 July 2013. First published online 14 May 2014.

\section{Introduction}

Protecting large carnivores in the face of increasing 1 human populations, agricultural expansion and overuse of natural resources is one of the biggest conservation challenges. Communities in the vicinity of wildlife parks and sanctuaries are reliant upon livestock for their livelihoods, resulting in increasing numbers of livestock even within protected areas (Mishra et al., 2004). The resulting distorted relative proportions of wild and domestic prey may trigger attacks by carnivores on domestic stock (Mishra et al., 2003). The economic cost of depredation on livestock results in a negative attitude towards carnivores (Treves et al., 2009) and further aggravates humancarnivore conflicts (Graham et al., 2005). Large carnivores also attack humans occasionally (Conover et al., 1995; Packer et al., 2005; Lodhi, 2007; White \& Gehrt, 2009), resulting in injury and sometimes loss of life. The nature and extent of such conflicts seem to be more severe in South Asia (Mishra, 1997; Mishra et al., 2003; Ogra, 2008) as a result of limited availability and sharing of resources.

A typical example of human-carnivore conflict in Pakistan involves the common leopard Panthera pardus. Although highly adaptable and widespread in Africa and Asia (Nowell \& Jackson, 1996), the leopard is threatened in Pakistan (Sheikh \& Molur, 2004). It has flexible habitat preferences, occupying dense forest, rock and scrub, grasslands and mountain cliffs where sufficient prey is available (Prater, 1993; Nowell \& Jackson, 1996; Al-Johany, 2007). In Pakistan it is confined to Himalayan forest regions up to the limit of the tree line, and lower-altitude valleys in arid mountainous regions. It also inhabits broken, hilly terrain with Acacia scrub forest cover throughout Waziristan, Baluchistan and Sindh Kohistan (Roberts, 1997). The forested areas of the Himalaya, particularly the Galliat in the Khyber Pakhtunkhwa province and Neelam Valley in the state of Azad Jammu and Kashmir, are probably the main strongholds of the leopard in Pakistan. Elsewhere, the common leopard is rare, having been hunted almost to extinction by trophy hunters and in retaliatory killings by local people (Roberts, 1997).

Since 2000 there have been increasing reports of leopard-human conflict in the forested areas of the Himalaya, with casualties on both sides. The Khyber Pakhtunkhwa Wildlife Department reported 142 livestock losses attributed to common leopards in the Ayubia National Park (Lodhi, 2007), and 363 livestock losses were 


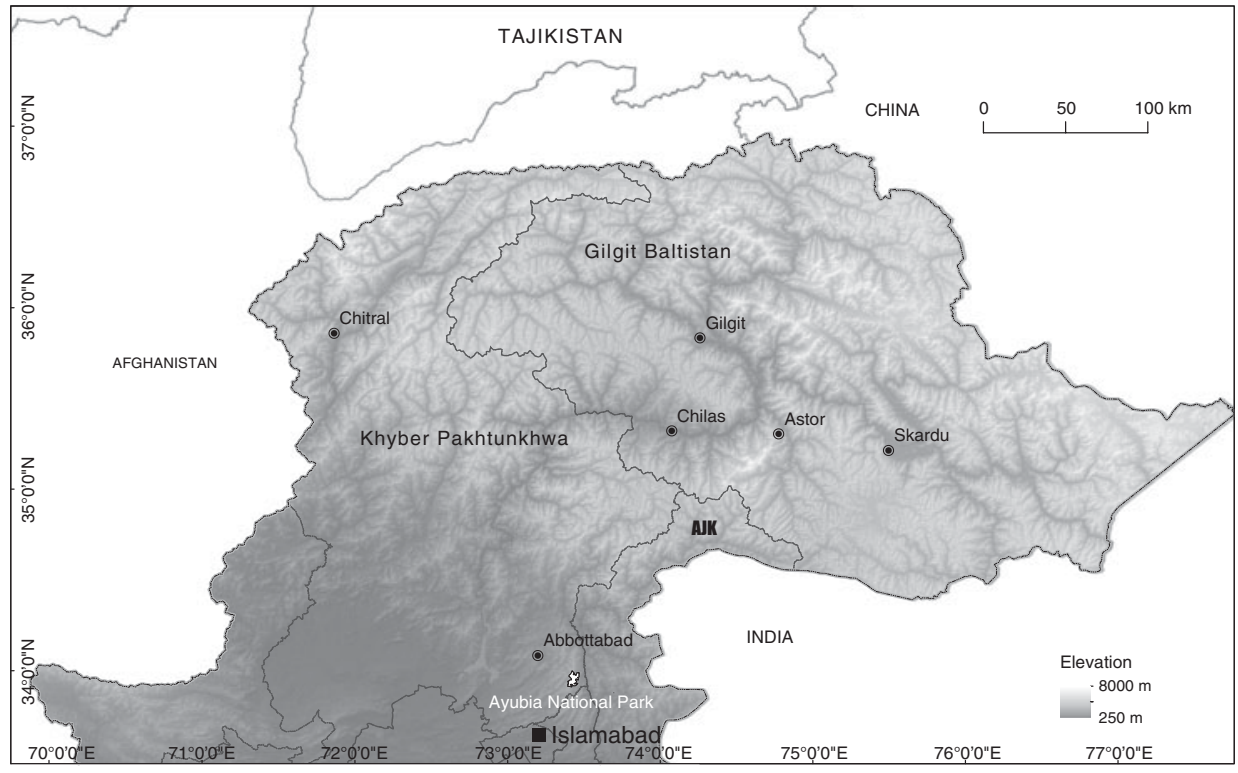

FIG. 1 North-east Pakistan, showing the location of Ayubia National Park. reported from the Machiara National Park, Azad Jammu and Kashmir (Dar et al., 2009) during a 3-year period, indicating that the common leopard is the major livestock predator (90\%) in the Park. Kabir \& Waseem (2010) documented 72 livestock losses from the Pir Lasora National Park, Azad Jammu and Kashmir, comprising goats Capra hircus (46\%), domestic dogs Canis lupus familiaris (32\%), sheep Ovis aries (17\%) and cows Bos taurus (4\%). In addition to depredation on domestic livestock there has been an increase in the number of reports of fatal attacks on humans by leopards.

The Khyber Pakhtunkhwa Wildlife Department has organized a number of consultative workshops to help formulate an effective strategy for conservation of the common leopard in Pakistan and address the various challenges involved. There is a dearth of information about the population and ecology of leopards in Pakistan, and wildlife managers and conservationists have divergent opinions. Local communities attribute the increase in human-leopard conflict to an increasing leopard population, and propose trophy hunting as a solution (Lodhi, 2007). However, wildlife managers argue that the assumption of population increase is not substantiated by credible data and that reports of livestock predation are exaggerated, because the leopards have sufficient wild prey available in the form of rhesus macaques Macaca mulatta.

An accurate assessment of livestock predation is necessary to measure the scale of the human-leopard conflict and design appropriate management strategies. Here we apply a DNA-based diet analysis technique (Shehzad et al., 2012b) to a population of the common leopard in Ayubia National Park. Our findings facilitate understanding of the feeding ecology of the common leopard and its conflict with the local community and will inform efforts to mitigate this conflict.

\section{Study area}

Ayubia National Park $\left(33 \mathrm{~km}^{2}\right)$ was established in 1984 and is surrounded by $150 \mathrm{~km}^{2}$ of reserved forests (Fig. 1). It hosts c. 200 species of birds, 31 species of mammals, 16 species of reptiles and three species of amphibians (Farooque, 2007). The creation of the Park has facilitated the recovery of a number of species, including the common leopard, which was close to extinction in the Galiat region and adjacent areas by the early 1980 (Lodhi, 2007).

\section{Methods}

We analysed leopard diet by characterizing the prey DNA present in faecal samples after amplification of a diagnostic fragment and sequencing of polymerase chain reaction (PCR) products, using next-generation sequencing. This provides diet information without any a priori knowledge about the prey and is a cost-effective method as millions of readings can be generated from a single sequencing run. This method has several advantages over classical microscopy, which requires substantial skill and time and is prone to misidentification in the case of closely related species (Pompanon et al., 2012).

Faecal samples were collected by three experienced researchers and nine park guards. The guards were experienced in identifying leopard sign, including faeces, and they also received 1 day of training before the survey. Three teams of 3-4 persons were mobilized every day during 25 June-6 July 2008 and a total of 27 transects of $2-7 \mathrm{~km}$ were established in the Park and surrounding reserve forest for the collection of samples. Movement in the area is restricted to available tracks but transects were placed to maximize the spatial coverage of samples. The age of all samples collected was estimated based on their physical 
appearance. One hundred and eleven putative leopard faecal samples were collected.

All DNA extraction was performed in a laboratory dedicated to the extraction of degraded DNA. Total DNA was extracted from c. $15 \mathrm{mg}$ of faeces, using the DNeasy Blood and Tissue Kit (QIAgen GmbH, Hilden, Germany), following the manufacturer's instructions, with a slight modification as described by Shehzad et al. (2012b). Blank extractions were performed to monitor any contamination.

The sequence analysis and taxon assignation were done using OBITools, as described in Shehzad et al. (2012a, b). Full details of the primer design and DNA sequencing can be found in Supplementary Material 1 and Supplementary Table Si.

To evaluate adequacy of sample size for characterizing leopard diet we constructed a species accumulation curve and extrapolated the species richness, using the specpool function in $R$ (R Development Core Team, 2012). This function estimates the number of undetected species and adds them to the observed species richness (Palmer, 1990; Colwell \& Coddington, 1994).

\section{Results}

Of 111 putative leopard faecal samples collected from Ayubia National Park and surroundings, $43 \%$ were $\geqslant 1$ month old and the remainder were fresh samples. Sixty (54\%) samples were identified as of common leopard, using the common leopard specific primer pair (PantF/PantR), and selected for further experimentation. After assembling the forward and reverse reads and filtering for primers and tags we obtained a total of 652,090 sequences, corresponding to 36,929 different sequences (DRYAD, 2014). The removal of sequences shorter than $60 \mathrm{bp}$ and with a total count of $<100$ reduced the dataset to 150 sequences. The next step aimed to remove sequences corresponding to PCR errors. We used the obiclean algorithm, which relies on a directed acyclic graph build for each PCR, where vertices are sequences and edges link two vertices differing only by one substitution or insertion/deletion (De Barba et al., 2014). True sequences are assumed to be 'head' (i.e. the most frequent sequence among all sequences that can be linked together) or 'singleton' (i.e. a sequence with no variant differing by a single indel or substitution). All other sequences $(n=94)$ were removed from the dataset. We also removed 41 sequences with a total count among all samples of $<750$. This threshold was determined to avoid having the same species identified twice in the result list (a first identification corresponding to the true sequence and a second one corresponding to an artifactual sequence). Finally, three sequences without a perfect identity corresponding to the reference database were also removed, resulting in 12 (including one leopard and 11 prey sequences) molecular operational taxonomic units (Blaxter, 2004).
Supplementary Table S2 presents an overview of sequence counts from next-generation sequencing initial files at different stages of the analysis. The filtered data are available at DRYAD (2014). All faecal samples identified as common leopard, using the common leopard specific primer pair (PantF/PantR), were further confirmed by the sequencing, and common leopard sequences were observed in all samples, with an overall relative frequency of 0.16 . This means that the $2 \mu \mathrm{M}$ concentration of blocking oligonucleotide used in this experiment reduced but did not completely prevent amplification of the common leopard sequence. Only common leopard DNA was amplified from three samples, with no information about prey items. Eleven prey taxa were identified in the remaining 57 samples. Three prey items were identified in one sample, two prey items in seven samples, and single prey items in 49 samples. Based on the frequency of occurrence of prey items in the 57 faecal samples, the domestic goat predominated the diet (64.9\%), followed by $\operatorname{dog}(17.5 \%)$ and cow (12.3\%). Domestic animals (goat, dog, cow, water buffalo Bubalus bubalis, horse Equus caballus and sheep) occurred in 54 of 57 samples, corresponding to a frequency of occurrence of 0.95 , and five samples contained two items of domestic prey. Fig. 2 illustrates the relative frequencies of prey items present in the leopard's diet.

Main prey items (goat, dog, cow, buffalo), with cumulative frequency of $85 \%$, were detected in the first four samples. A species accumulation curve with 100 permutations reached a species richness of 10 at sample 48 , so there was an addition of only one species in the remaining 12 samples. The extrapolated species richness was $13.67 \pm S D 3.48$, which is close to the observed number of species (11). On this basis we believe that the sample size was adequate to characterize the diet of common leopards, although additional samples could reveal some occasionally or opportunistically eaten species.

\section{Discussion}

Analysis of faecal samples by combining universal primers for vertebrates (plus a blocking oligonucleotide specific to predator DNA) with next-generation sequencing revealed the diet of the common leopard in Pakistan. In 57 samples we identified a total of 11 prey species. Our results confirm that the common leopard is a generalist predator, consuming a wide variety of prey, including ungulates, carnivores, rodents, birds and fish.

Throughout the leopard's range there has been a shift in its prey choices towards livestock (Judas et al., 2006; Spalton \& $\mathrm{Al} \mathrm{Hikmani,} \mathrm{2006).} \mathrm{Circa} \mathrm{95 \%} \mathrm{of} \mathrm{the} \mathrm{leopard} \mathrm{prey} \mathrm{items}$ in Ayubia National Park are domestic animals and there are a number of possible reasons for such a high predation rate. Firstly, the lack of natural prey in the Park provokes leopard attacks on domestic stock. Secondly, poor husbandry and 


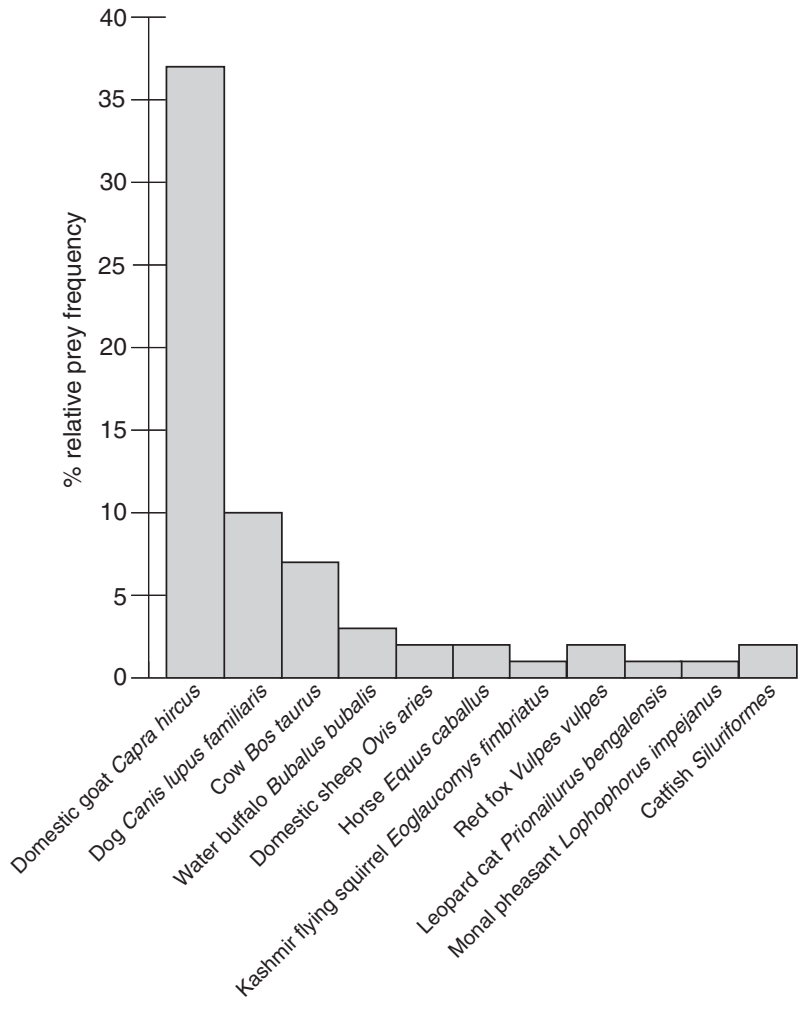

FIG. 2 Relative frequency of prey items in the diet of the common leopard Panthera pardus in Ayubia National Park, Pakistan (Fig. 1).

penning of livestock make domestic animals more vulnerable to leopard attacks. Thirdly, seasonal factors affect the susceptibility of livestock to predation; the sampling for this study was carried out during the summer, when most of the livestock is sent to pasture for grazing and is mostly unattended. Grazing livestock is occasionally guarded by dogs, which are the second-most common leopard prey species in summer. We also found leopard cat Prionailurus bengalensis, red fox Vulpes vulpes and Kashmir flying squirrel Eoglaucomys fimbriatus in the leopard diet, which have not been reported previously as leopard prey. Among the 11 taxa predated by leopards in Ayubia only one species, the Kashmir flying squirrel, is categorized as Vulnerable in Pakistan (based on national assessment of mammals in Pakistan; Sheikh \& Molur, 2004). This species was present in a single sample, indicating that it is not under significant predation pressure from leopards, although it is heavily predated by the leopard cat in the same area (Shehzad et al., 2012b). As our results are based on summer diet they may not reflect the complete diet profile of the leopard in the area surveyed. Future work based on samples collected year-round could reveal more prey taxa in the leopard diet.

The rhesus macaque is common in Ayubia National Park and is generally believed to constitute a major part of the leopard diet there (Lodhi, 2007). However, our findings do not support this perception as we did not find any monkey sequences in the leopard faeces despite the fact that our reference database contained several exemplars. There are conflicting reports about leopard predation on primates from different parts of its range in Asia and Africa (Kummer et al., 1981; Cowlishaw, 1994; Isbell, 1994; Nowell \& Jackson, 1996; Zuberbühler \& Jenny, 2002; Hayward et al., 2006). Leopards have disparate preferences for different primate species, and scientific data indicate that leopards rarely prey on rhesus macaque (Mukherjee \& Mishra, 2001). We therefore believe that reports (Lodhi, 2007) of leopard predation on rhesus macaque in Ayubia are based on anecdotal information and misconception because the monkeys are abundant in the Park.

Our findings indicate that the leopard population in Ayubia is sustained by domestic animals. Actual loss of livestock may be even higher than that observed in the diet analysis because leopards are considered to be surplus killers and have been reported to kill up to 22 sheep in a single attack (Sangay \& Vernes, 2008). This level of predation can have adverse effects on the local economy and cause conflict between the leopard and the local community. Mitigation of this conflict is an important factor in leopard conservation in the Park.

We recommend a two-step strategy to engage the local community in conservation efforts. Immediate measures could include the introduction of incentives to foster a greater acceptance of the predator, such as support for livestock insurance (Mishra et al., 2003), vaccination programmes (Nawaz, 2009) and improving livestockguarding facilities (Dar et al., 2009). Such incentives could be subsidized from the revenue generated by Park entry fees and associated tourism.

Measures to curtail predation can also help reduce conflict and can be complemented by incentive programmes. For example, improvements in penning and guarding facilities can result in significant reductions in livestock losses to predation. Increased vigilance at night and guarding livestock during grazing can reduce the risk of leopard attacks (Dar et al., 2009).

A second step should focus on the reintroduction and protection of wild prey in the Park. Depletion of natural prey as a result of poaching is the main reason for the leopard's dependence on livestock for food and is thus a major source of conflict. Reintroduction of wild prey species such as goral Naemorhedus goral and musk deer Moschus moschiferus could help resolve these issues in the longer term. The Wildlife Department's existing captive facility at Nathia Gali could be used for breeding of potential prey species before release in the wild. However, such efforts would only be successful if poaching of these species in the Park and surrounding areas could be curbed through strict law enforcement.

We hope that our results will stimulate the implementation of such a conservation strategy and ensure the 
survival of the leopard population in Pakistan. The results of our study were shared informally with the Wildlife Department and conservation organizations working in the area, which promoted a realization that communities' concerns over livestock predation are real and significant. Consequently, programmes have been initiated to compensate communities for their losses.

\section{Acknowledgements}

The authors are grateful to Alice Valentini, Christian Miquel, Carole Poillot, Delphine Rioux and Ludovic Gielly for their valuable comments and suggestions throughout this study. Wasim Shehzad and Tiayyba Riaz were supported by $\mathrm{PhD}$ scholarships from the Higher Education Commission of the Government of Pakistan. The Khyber Pakhtunkhwa Wildlife Department, Himalayan Wildlife Foundation and Snow Leopard Trust organized sample collections in Ayubia National Park.

\section{References}

Al-Johany, A.M.H. (2007) Distribution and conservation of the Arabian leopard Panthera pardus nimr in Saudi Arabia. Journal of Arid Environments, 68, 20-30.

Bellemain, E., Carlsen, T., Brochmann, C., Coissac, E., Taberlet, P. \& Kauserud, H. (2010) ITS as an environmental DNA barcode for fungi: an in silico approach reveals potential PCR biases. BMC Microbiology, 10, 189-197.

Blaxter, M.L. (2004) The promise of a DNA taxonomy. Philosophical Transactions of the Royal Society of London Series B, 359, 669-679.

Brownstein, M.J., Carpten, J.D. \& Smith, J.R. (1996) Modulation of non-templated nucleotide addition by Taq polymerase: primer modifications that facilitate genotyping. BioTechniques, 20, 1004-1010.

Colwell, R.K. \& Coddington, J.A. (1994) Estimating terrestrial biodiversity through extrapolation. Philosophical Transactions of the Royal Society of London Series B, 345, 101-118.

Conover, M.R., Pitt, W.C., Kessler, K.K., DuBow, T.J. \& SAnBorn, W.A. (1995) Review of human injuries, illnesses, and economic losses caused by wildlife in the United States. Wildife Society Bulletin, 23, 407-414.

Cowlishaw, G. (1994) Vulnerability to predation in baboon populations. Behaviour, 131, 293-304.

Dar, N.I., Minhas, R.A., Zaman, Q. \& Linkie, M. (2009) Predicting the patterns, perceptions and causes of human-carnivore conflict in and around Machiara National Park, Pakistan. Biological Conservation, 142, 2076-2082.

De Barba, M., Miquel, C., Boyer, F., Mercier, C., Rioux, D., Coissac, E. \& TABerlet, P. (2014) DNA metabarcoding multiplexing and validation of data accuracy for diet assessment: application to omnivorous diet. Molecular Ecology Resources, 14, 306-323.

DrYAD (2014) Http://datadryad.org/resource/doi:10.5061/dryad.87rm1

Farooque, M. (2007) Management Plan of Ayubia National Park. Khyber Pakhtunkhwa Wildlife Department, Peshawar, Pakistan.

Ficetola, G.F., Coissac, E., Zundel, S., Riaz, T., Shehzad, W., BESSIÈre, J. et al. (2010) An in silico approach for the evaluation of DNA barcodes. BMC Genomics, 11, 434-443.
Graham, K., Beckerman, A.P. \& Thirgood, S. (2005) Human-predator-prey conflicts: ecological correlates, prey losses and patterns of management. Biological Conservation, 122, 159-171.

Hayward, M.W., Henschel, P., O’Brien, J., Hofmeyr, M., Balme, G. \& Kerley, G.I.H. (2006) Prey preferences of the leopard (Panthera pardus). Journal of Zoology, 270, 298-313.

Isbell, L.A. (1994) Predation on primates: ecological patterns and evolutionary consequences. Evolutionary Anthropology, $3,61-71$.

Judas, J., Paillat, P., Khoja, A. \& Boug, A. (2006) Status of the Arabian leopard in Saudi Arabia. Cat News, Special Issue 1, 11-19.

Kabir, M. \& Waseem, M. (2010) Human-Leopard Conflict Assessment in Pir Lasora National Park, Kotli, Azad Jammu and Kashmir, Pakistan. WWF-Pakistan, Lahore, Pakistan.

Kummer, H.A., Banaja, A.A., Aro-Khatwa, A.N. \& Grandour, A.M. (1981) A survey of hamadryas baboons in Saudi Arabia. In Fauna of Saudi Arabia, Volume 3 (eds W. Wittmer \& W. Biittiker), pp. 441-471. Natural History Museum, Basel, Switzerland.

Lodhi, A. (2007) Conservation of leopards in Ayubia National Park, Pakistan. MSc thesis. The University of Montana, Missoula, USA.

Magnuson, V.L., Ally, D.S., Nylund, S.J., Karanjawala, Z.E., RaYman, J.B., Knapp, J.I. et al. (1996) Substrate nucleotidedetermined non-templated addition of adenine by Taq DNA polymerase: implications for PCR-based genotyping and cloning. BioTechniques, 21, 700-709.

Mishra, C. (1997) Livestock depredation by large carnivores in the Indian trans-Himalaya: conflict perceptions and conservation prospects. Environmental Conservation, 24, 338-343.

Mishra, C., Allen, P., McCarthy, T., Madhusudan, M.D., Bayarjargal, A. \& Prins, H.H.T. (2003) The role of incentive programs in conserving the snow leopard. Conservation Biology, $17,1512-1520$.

Mishra, C., Van Wieren, S.E., Ketner, P., Heitkonig, I.M.A. \& PrIns, H.H.T. (2004) Competition between domestic livestock and wild bharal Pseudois nayaur in the Indian Trans-Himalaya. Journal of Applied Ecology, 41, 344-354.

Muknerjee, S. \& Mishra, C. (2001) Predation by leopard Panthera pardus in Majhatal Harsang Wildlife Sanctuary, Western Himalaya. Journal of the Bombay Natural History Society, $98,267-268$.

Nawaz, M.A. (2009) Snow Leopard-Friendly Vaccination Program Set to Expand in Pakistan. Snow Leopard Trust, Seattle, USA.

Nowell, K. \& JACKsON, P. (eds) (1996) Wild Cats: Status Survey and Conservation Action Plan. IUCN, Gland, Switzerland.

OGRA, M.V. (2008) Human-wildlife conflict and gender in protected area borderlands: a case study of costs, perceptions, and vulnerabilities from Uttarakhand (Uttaranchal), India. Geoforum, 39, 1408-1422.

Packer, C., Ikanda, D., Kissui, B. \& Kushnir, H. (2005) Lion attacks on humans in Tanzania-Understanding the timing and distribution of attacks on rural communities will help to prevent them. Nature, 436, 927-928.

Palmer, M.W. (1990) The estimation of species richness by extrapolation. Ecology, 71, 1195-1198.

Pompanon, F., Deagle, B.E., Symondson, W.O.C., Brown, D.S., JARMAN, S.N. \& TABERLET, P. (2012) Who is eating what: diet assessment using next generation sequencing. Molecular Ecology, 21, 1931-1950.

Prater, S.H. (1993) The Book of Indian Animals. Oxford University Press, New Delhi, India.

R Development Core Team (2012) R: A Language and Environment for Statistical Computing. R Foundation for Statistical Computing, Vienna, Austria. 
Riaz, T., Shehzad, W., Viari, A., Pompanon, F., Taberlet, P. \& CoISSAC, E. (2011) ecoPrimers: inference of new DNA barcode markers from whole genome sequence analysis. Nucleic Acids Research, 39, e145.

Roberts, T.J. (1997) Mammals of Pakistan. Oxford University Press, Karachi, Pakistan.

Sangay, T. \& Vernes, K. (2008) Human-wildlife conflict in the Kingdom of Bhutan: patterns of livestock predation by large mammalian carnivores. Biological Conservation, 141, $1272-1282$.

Shehzad, W., McCarthy, T.M., Pompanon, F., Purevjav, L., Coissac, E., Riaz, T. \& Taberlet, P. (2012a) Prey preference of snow leopard (Panthera uncia) in South Gobi, Mongolia. PLOS ONE, 7(2), e32104.

Shehzad, W., Riaz, T., Nawaz, M.A., Miquel, C., Poillot, C., SHAH, S.A. et al. (2012b) Carnivore diet analysis based on next-generation sequencing: application to the leopard cat (Prionailurus bengalensis) in Pakistan. Molecular Ecology, 21, 1951-1965.

Sheikh, K.M. \& Molur, S. (eds) (2004) Status and Red List of Pakistan's Mammals. Based on the Conservation Assessment and Management Plan. IUCN Pakistan, Islamabad, Pakistan.

Spalton, J.A. \& Al Hikmani, H.M. (2006) The leopard in the Arabian Peninsula-distribution and subspecies status. Cat News, Special Issue 1, 4-8.

Treves, A., Wallace, R.B. \& White, S. (2009) Participatory planning of interventions to mitigate human-wildlife conflicts. Conservation Biology, 23, 1577-1587.

Valentini, A., Miquel, C., Nawaz, M.A., Bellemain, E., Coissac, E., Pompanon, F. et al. (2009) New perspectives in diet analysis based on DNA barcoding and parallel pyrosequencing: the trnL approach. Molecular Ecology Resources, 9, 51-60

Vestheim, H. \& Jarman, S.N. (2008) Blocking primers to enhance PCR amplification of rare sequences in mixed samples-a case study on prey DNA in Antarctic krill stomachs. Frontiers in Zoology, 5.

White, L.A. \& Gehrt, S.D. (2009) Coyote attacks on humans in the United States and Canada. Human Dimensions of Wildlife, $14,419-432$.

Zuberbühler, K. \& Jenny, D. (2002) Leopard predation and primate evolution. Journal of Human Evolution, 43, 873-886.

\section{Biographical sketches}

WASIM SHEHZAD's research interests cover ecological studies exploiting DNA from non-invasive sampling, and applying this approach to biodiversity surveys and the identification of elusive and threatened species. Ali NAWAZ is interested in molecular and field studies for natural resource management, and has been focusing on understanding ecology, co-existence and conservation issues of the carnivore community in Pakistan. François POMPANON studies adaptation through population genomics approaches and develops molecular markers for describing biodiversity. ERIC COISSAC and Tiаутва Riaz are involved in developing the DNA metabarcoding approach, focusing on the bioinformatics aspects. SAFDAR ALI SHAH is experienced in collaborative management of biodiversity and its sustainable use through community participation. PIERRE TABERLET is interested in conservation genetics and develops molecular methods (including metabarcoding) for studying non-invasive samples. 